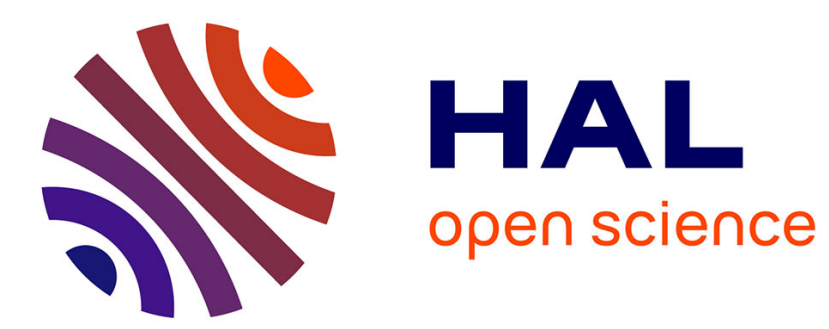

\title{
Laminar, transitional, and turbulent flows in rotor-stator cavities
}

\author{
Brian Launder, Sébastien Poncet, Eric Serre
}

\section{To cite this version:}

Brian Launder, Sébastien Poncet, Eric Serre. Laminar, transitional, and turbulent flows in rotor-stator cavities. Annual Review of Fluid Mechanics, 2010, 42 (1), pp.229-248. 10.1146/annurev-fluid-121108145514. hal-00678846

\section{HAL Id: hal-00678846 https://hal.science/hal-00678846}

Submitted on 14 Mar 2012

HAL is a multi-disciplinary open access archive for the deposit and dissemination of scientific research documents, whether they are published or not. The documents may come from teaching and research institutions in France or abroad, or from public or private research centers.
L'archive ouverte pluridisciplinaire HAL, est destinée au dépôt et à la diffusion de documents scientifiques de niveau recherche, publiés ou non, émanant des établissements d'enseignement et de recherche français ou étrangers, des laboratoires publics ou privés. 


\title{
Laminar, Transitional and Turbulent Flows in Rotor-Stator Cavities
}

\author{
by \\ Brian Launder ${ }^{a}$, Sébastien Poncet $^{b}$ and Eric Serre ${ }^{b}$ \\ ${ }^{a}$ School of Mechanical, Aerospace \& Civil Engineering \\ The University of Manchester \\ PO Box 88, Manchester M60 1QD, UK \\ brian.launder@manchester.ac.uk \\ Personal webpage: http://www.mace.manchester.ac.uk/aboutus/staff/academic/profile/?staffId=176 \\ ${ }^{b}$ Laboratoire M2P2, UMR 618 CNRS - Aix-Marseille Université \\ poncet@L3m.univ-mrs.fr \\ Personal webpage: http://13mgp.13m.univ-mrs.fr/site/SitePersoPoncet/ \\ Eric.Serre@L3m.univ-mrs.fr \\ Personal webpage: http://13mgp.13m.univ- \\ mrs.fr/virtual.php?page site=site/Intranet/../SERREericperso/SERREEricwebperso2.htm
}

\begin{abstract}
The paper reviews the range of flows that may be created within thin cylindrical or annular cavities due to the rotation of one of the confining disks. At low Reynolds numbers the rotation gives rise to an axisymmetric, radially-outward motion near the rotor with a return flow along the stationary disk. As the Reynolds number is raised this base flow gives way to a shear flow populated by discrete vortices, whether of cylindrical or spiral form, near both the rotating and stationary disks. At Reynolds numbers high enough for turbulent flow to occur, in the $20^{\text {th }}$ Century both experimental and computational studies treated the flow as axisymmetric and steady. Recent research has shown, however, that complex organized structures also persist in the turbulent regime.
\end{abstract}

\section{Key Words}

Rotor-stator cavity, Ekman layer, three-dimensional boundary layer, crossflow instability, RANS closures, LES

\section{Acronyms list}

ASM : Algebraic Stress Models

DNS: Direct Numerical Simulation

LDA/V: Laser Doppler Anemometry/Velocimetry

LES: Large Eddy Simulation

TCL: Two-Component Limit

(U)RANS: (Unsteady) Reynolds-Averaged Navier-Stokes

\section{Summary Points list}

1. The laminar base flow is axisymmetric and three-dimensional due to the recirculation in the meridional plane created by the centrifugal force associated with the rotation of the disk. In this plane, the flow is predominantly radial within the boundary layers along the disks. 
2. The stability of the flow is governed by the boundary layers and is closely connected with that of a single infinite rotating disk.

3. The cross-flow instability appearing as travelling circular or spiral rolls governs the transition scenario to turbulence.

4. Organized vortex structures are also present in the turbulent flow regime. Those occurring close to the discs are well-resolved by LES computations.

5. Large-scale vortex structures in the core of the flow have not been satisfactorily captured by either URANS or LES computations.

6. The circumferentially- and temporally-averaged flow features are, for the most part, satisfactorily resolved by RANS modelling though the width of agreement understandably increases with the level of closure. Best agreement reported to date is with a second-moment closure satisfying the TCL constraint.

\section{Future Issues}

1. Our knowledge of the different scenarios for transition must be further extended. In particular, the role of the cross-flow instability on turbulence breakdown needs to be clarified.

2. Efficient control strategies to delay or promote the transition to turbulence in the disk boundary layers remain to be developed.

3. An urgent question to resolve is whether, in turbulent flow, the failure to reproduce numerically the permanent large-scale structures is due to inadequate numerical modelling or whether the "failure" arises from relatively small departures of the actual experiments from the idealized test-case configurations.

4. Numerical modelling must be improved to facilitate the exploration via LES both of industrial configurations and physically more complex flows addressing, in particular, higher rotation rates, appreciable density variations associated to large temperature differences and finally more realistic geometries.

\section{Related Resources}

1. Greenspan HP. 1990. The Theory of Rotating Fluids. Breukelen Press, Brookline

2. Owen JM, Rogers RH. 1989. Flow and Heat Transfer in Rotating-Disc Systems. Vol. 2: Rotating cavities. Ed. WD Morris, New-York: Wiley \& Sons Inc.

3. School of Mechanical, Aerospace \& Civil Engineering. Website of the Turbulence Mechanics SIG: http://tmgflows.mace.manchester.ac.uk/\#rotatingpassages.

4. IRPHE / Geophysical and Rotating flows research team: https://www.irphe.univmrs.fr/ legal/ROT.html

5. FAST / Turbulence en rotation: http://www.fast.u-psud.fr/ moisy/turbrot/turb_fr.php

6. LIMSI / Groupe Convection et Rotation: http://www.limsi.fr/Scientifique/coro/galerie 


\section{INTRODUCTION}

Confined cavity flows between two coaxial disks, one or both of which are rotating, and closed by a cylindrical sleeve or shroud, are both rich in the flow phenomena they create and the range of industrial flows with which they are linked. The sheer diversity of the resultant flows has required, however, that the present review limits attention to cases where one of the disks is at rest, where the inter-disk spacing is small compared with the disk radius and where there is no through-flow. Some impression of the variety of flow structures arising with other boundary conditions may, however, be gained from the book by Owen \& Rogers (1989), the review of engineering disk cavities by Owen (2000) and, from specific studies: for example, by Escudier (1984) and Serre \& Bontoux (2001) for tall cavities, Abrahamson et al. (1991) and Humphrey et al. (1995) for co-rotating disks and Moisy et al. (2004) for counter-rotating disks.

At the rotating disk itself the no-slip condition ensures that the motion is purely circumferential. For the narrow cavities under consideration, however, the radial pressure gradient induced by the disk's rotation varies little between the disks, being determined by the smaller circumferential velocities prevailing in the core region away from the disk surfaces. In the strongly swirling flow adjacent to the rotating disk, this pressure gradient is insufficient to prevent a radial outflow which may exceed $10 \%$ of the local disk speed. Thus, as first noted by Ekman (1902) in connection with the atmospheric boundary layer, the mean velocity vector undergoes skewing as one moves away from the wall. This skewing is an essential contributor to the variety of the flow patterns that may result.

Analogously, close to the stationary disk, the swirling flow is retarded by viscous or turbulent stresses below that in the core. Because the pressure gradient is then too strong for radial equilibrium, the fluid moves radially inwards. On the shroud, because the inter-disk spacing is small, the classical Taylor-Görtler instability arising from shear flow over curved 
surfaces may be of only secondary importance. The shroud does, however, additionally contribute to the flow complexity by provoking the separation of the rotor boundary layer and causing this flow to impinge on the stator wall.

Concerning the remainder of the review, Sections 2 and 3 below define the geometry, the terminology adopted, and the important dimensionless parameters governing the flow together with a parametric map of the principal flow regimes. The main focus of the paper is however on the instability patterns that are created leading to transition and eventually to turbulence and on the turbulent flow regime itself. These form the subjects of Sections 4 and 5 in which the flow structures are principally conveyed by photographs or equivalent computer graphics.

\section{GEOMETRY AND CHARACTERISTIC FLOW PARAMETERS}

The geometrical model of the rotor-stator cavity comprises two smooth coaxial parallel disks enclosing either a cylindrical or an annular domain (Figure 1) of internal and external radii $a$ and $b$, respectively. For cylindrical cavities $(a=0)$ it includes the rotation axis. The upper disk of the cavity is at rest (stator) while the lower disk rotates at uniform angular velocity $\Omega$ (rotor). The flow domain is bounded by one or two cylinders of height $h$, the inner one (the hub), attached to the rotor, being rotating while the outer one (the shroud), attached to the stator, is at rest. In practice, there is a small radial clearance, $j$, between the shroud and the rotor which exerts negligible effects for the flows considered here.

This geometry is fully characterized by two geometrical parameters formed from the combination of the three independent length scales, $a, b, h$; these are usually chosen as the aspect ratio of the cavity $G \equiv h / b$ and the curvature parameter $R_{m} \equiv(b+a) /(b-a)$. For a cylindrical cavity, $R_{m}=1$. The present review limits attention to the case of radially elongated cavities $(G<1)$ because of their relevance to cavities in gas-turbine systems. 
The structure of the base flow is characterized by some global parameter, which may be taken as a Reynolds number, $\operatorname{Re}_{h} \equiv(h / \delta)^{2}$ which scales the boundary layer thicknesses of characteristic length $\delta \equiv(v / \Omega)^{1 / 2}$ with respect to the cavity height, $h$. Stability analysis requires two local control parameters, Ro and $\mathrm{Re}_{r}$, due to the flow's radial inhomogeneity. The local Rossby number is defined, following Lingwood (1997), as: Ro $\equiv\left(\Omega_{f}-\Omega\right) / \widetilde{\Omega}$ where $\Omega_{f}$ is the fluid's angular velocity in the core, and $\widetilde{\Omega}$ is a reference rotation rate of the system

$$
\tilde{\Omega} \equiv\left(\Omega_{f}+\Omega\right) / 4+\sqrt{\left(\left(\Omega_{f}+\Omega\right) / 4\right)^{2}+\left(\Omega_{f}-\Omega\right)^{2} / 2} \text {. This parameter compares the (nonlinear) inertial }
$$

effects to the (linear) Coriolis force, so that when Ro goes to zero, the flow solution becomes linear. The local Reynolds number is defined as the dimensionless radius $\operatorname{Re}_{r} \equiv(r / \delta)$. In the literature of finite disk systems, the Reynolds number can alternatively be based on the external radius of the cavity, $\mathrm{Re} \equiv \Omega b^{2} / v$ which is easy to obtain and gives an upper bound to the (square of the) local Reynolds number, $\operatorname{Re}_{r}$. The two global parameters are linked by the relation $\operatorname{Re}_{h}=\operatorname{Re} . G^{2}$.

\section{BASE FLOW}

The laminar base flow is axisymmetric and three-dimensional due to the circulation in the meridional plane $(r, z)$ created by the centrifugal force associated with the rotation of the disk. In this plane, the flow is essentially radial within the boundary layers along the disks.

In infinite cavities $(G \rightarrow 0)$, the base flow is governed purely by the global parameter $\operatorname{Re}_{h}$. For small $\mathrm{Re}_{h}$, the disk boundary layers merge and the basic state is purely viscous. At large $\mathrm{Re}_{h}$, Mellor et al. (1968) (numerically) and Kreiss \& Partner (1983) (analytically) have demonstrated that many similarity solutions exist. The two best known solutions are those obtained by Batchelor (1951) and Stewartson (1953) because of their relevance to flows observed in finite cavities. Batchelor (1951) showed that the two boundary layers are 
separated by a central core in solid-body rotation characterized by zero radial velocity $\left(V_{r}=0\right)$ and zero axial gradient of velocity as a direct consequence of the Taylor-Proudman theorem. The alternative solution proposed by Stewartson (1953) consists of a single boundary layer at the rotating disk and a core above it which does not rotate.

The main effect of finite-radius disks is that the boundary conditions are not compatible with self-similarity solutions though there may be qualitative resemblance far from the end walls, Brady \& Durlofski (1987). Indeed, these authors suggest that the base flow is completely determined by the radial boundary conditions and hence unique for a prescribed set of initial and boundary conditions. Thus, for sufficiently large $\operatorname{Re}_{h}$, a base flow qualitatively similar to the Batchelor solution occurs in closed geometries, whereas the Stewartson solution commonly appears in cavities with through-flow (Daily et al. 1964, Poncet et al. 2005b) or in open cavities (i.e. without a shroud).

In the limit $G<<1$, Daily \& Nece (1960) identify the four flow regimes shown in Figure 2. According to Dyment (1981), the boundary between Regimes I and II is given by $G \cdot \operatorname{Re}^{1 / 2}=\alpha$, where $\alpha$ is a constant greater than unity. In the turbulent regime, where the thickness of the disk boundary layers increases greatly, the transition from Regimes III and IV satisfactorily fits $G \cdot \operatorname{Re}^{1 / 5} \approx 0.5$. As reported by Cooper \& Reshotko (1975), many transitions may be observed between all these flow regimes: II $\rightarrow$ IV $\rightarrow$ III; II $\rightarrow$ I $\rightarrow$ III; IV $\rightarrow$ III as well as more expected transitions such as I $\rightarrow$ III or II $\rightarrow$ IV. The thickening of the boundary layers combined with the increasing value of the local Reynolds number $\operatorname{Re}_{r}$ when moving towards the periphery of the cavity explain the transition scenarios II $\rightarrow$ IV $\rightarrow$ III and II $\rightarrow$ I $\rightarrow$ III for a given set of parameters $(\operatorname{Re}, G)$.

By analogy with the similarity solution between infinite disks, flows in Regime II are usually referred to as of Batchelor type (Batchelor 1951). In the core, the normalized circumferential velocity component is used to define an entrainment coefficient $K \equiv \Omega_{f} / \Omega$. The 
departure of finite-disk flows from the similarity solutions (Zandbergen \& Dijkstra 1987) can be characterized by the resultant value of $K$. For infinite-disk cavities, laminar similarity solutions give a constant value, $K=0.313$, as obtained numerically by Pearson (1965) and theoretically by Rasmussen (1971). For finite-disk cavities, both the experimental measurements of Gauthier et al. (1999) in a cylindrical cavity $(G=0.0479)$ and the numerical results of Serre et al. (2001) in an annular cavity $\left(G=0.04, R_{m}=5\right)$ show that $K$ is an increasing function of the local radius $r$. Such behaviour underlines that the base flow features are not homogeneous in the radial direction and require, as noted in $\S 2$, local scaling parameters such as $\mathrm{Ro}$ and $\mathrm{Re}_{r}$. The rotor boundary-layer is usually termed (somewhat simplistically) the Ekman layer in reference to the linear similarity solution $(\mathrm{Ro}=0)$ of a fluid rotating over an infinite single disk rotating at the same speed (Ekman 1902). In that layer, the radial velocity component is positive $\left(V_{r}>0\right)$ and the circumferential component $V_{\theta}$ ranges between the local speed of the disk, $\Omega r$, and the core speed, $K \Omega r$. In a confined cavity, by continuity, as there is no radial flow in the central core, the radial outflow near the rotor is necessarily compensated by an inward radial flow along the stator. By analogy with the similarity solution of a rotating fluid over a single, infinite, stationary disk $(\mathrm{Ro}=1)$, this layer is termed the Bödewadt layer (Bödewadt, 1940). The flow in that layer is centripetal $\left(V_{r}<0\right)$ and $V_{\theta}$ ranges from $K \Omega r$ to 0 . The flow passes from the rotor to the stator boundary layer along the shroud. The fluid flows radially inwards along the stationary disk then returns near the axis or along the hub into the rotor boundary layer. The axial velocity component is almost zero in the whole cavity apart from these two regions very close to the confining cylinders or to the axis where the hub is absent.

Compared to the regimes with distinct boundary layers, relatively few investigations have been devoted to Regime I with merged boundary layers probably because of the difficulty of performing accurate measurements with such small clearances. The basic state is 
purely viscous. The radial velocity component exhibits an S-shaped profile with an outward flow along the rotor and an inward flow along the stator (Daily \& Nece 1960). Apart from very close to the disks, the tangential velocity component varies almost linearly with the axial coordinate. These flow regimes are termed "torsional Couette flows with merged boundary layers" (Cros \& Le Gal, 2002).

\section{INSTABILITIES AND TRANSITION}

As noted in \$2, the review focuses on the case of radially elongated cavities $(G<1)$ for otherwise the rotating disk has only a very small effect except within its vicinity and the flow stability is simply dominated by the boundary condition on the shroud. A large variety of structures can be observed according to the combination $(G, \Omega)$ as mapped by Schouveiler et al. (2001) (Figure 3).

The limiting case of very thin cavities, $G<<1$, has been little documented. The boundary layers merge (Cros \& Le Gal 2002) and the flow tends to be of torsional Couette type with features close to narrow-gap Taylor-Couette systems disk cavities. The literature is much more extensive for steady base flows of Batchelor type. The stability is primarily governed by the disk boundary-layers and the waves they support that can be locally analysed by reference to theoretical results from infinite disks. Nevertheless, the presence of the hub and the shroud can introduce other phenomena that may alter the global stability of the flow and lead to ingestion of large-amplitude disturbances into the disk boundary layers, causing major disruptions of the layer, possibly unrelated to the stability of the layer itself (Lopez \& Weidman, 1996). Such phenomena may be related to the flow adjacent to the confining cylinders being centrifugally unstable (Taylor-Görtler). They may also be linked to the formation of corner vortices at the junction between the disks and the sidewalls due to a 
combination of the bending vortex lines around the corners, and the horizontal diffusion of vorticity transporting the vortex lines radially in the sidewall layers.

\subsection{Type I and Type II Instabilities}

In the Batchelor regime, the stability of the flow is closely connected with that of the single-disk. Experimental, numerical and theoretical results display similar structures related to the two basic types of linear instability, referred to as Type I and Type II which can arise for different Rossby numbers ranging from $\mathrm{Ro}=-1$ (von Kármán flow) to $\mathrm{Ro}=1$ (Bödewadt flow) (see Faller 1991, Lingwood 1997, Serre et al. 2004). The linear theory of the Ekman flow $(\mathrm{Ro}=0)$ by Lilly (1966) shows that Type I is an inviscid instability related to an inflexion point in the profile of the radial velocity, similar to the Tollmien-Schlichting instability of the flat-plate boundary layer (Reed \& Saric 1989). The Type II instability is more specific to the rotating-disk boundary layer since it is related to the combined effects of Coriolis and viscous forces and occurs at a lower critical Reynolds number than the Type I.

In terms of the overall flow, these instabilities appear as a waviness of the streamlines but since the disturbances have vorticity they appear as travelling vortices rolling up around a circular or spiral axis when the basic flow is subtracted from the total. They may be characterized by four parameters: the wavelength, $\lambda$, the orientation of their wave fronts with respect to the geostrophic flow, $\varepsilon$, the frequency, $\sigma=2 \pi f$, and the azimuthal wavenumber, $\beta$. The spiral orientation, the radial $(k=2 \pi / \lambda)$ and azimuthal wave numbers are interrelated by: $\tan (\varepsilon)=\beta / k$. The wavelength $\lambda$ which depends on the boundary-layer characteristic length $\delta \equiv(v / \Omega)^{12}$ is smaller for Type I than for Type II, and their orientation, $\varepsilon$, is positive (Type I) or null or negative (Type II).

The properties of these instabilities are entirely defined for similarity solutions obtained for infinite disks (see Crespo del Arco et al. 2005). In the Batchelor regime, the characteristic 
parameters found in the two-disk problem (Itoh 1991) agree closely with the results for a single rotating disk with $\mathrm{Ro}=0.313$ (Pikhtov \& Smirnov 1992) corresponding to the asymptotic value of Ro for the Batchelor solution. The linear stability analysis of a Batchelor flow at $\mathrm{Re}_{\mathrm{h}}=1000$, Serre et al. (2004), found $\mathrm{Re}_{\mathrm{rcI}}=35$ and $\mathrm{Re}_{\mathrm{rcII}}=48$ in the stator-layer compared with $\operatorname{Re}_{\mathrm{rcI}}=100$ and $\mathrm{Re}_{\mathrm{rcI}}=280$ in the rotor layer.

Previous results cannot be directly extended to finite disks but provide good reference data. The radial confinement makes comparison with theoretical results difficult. There is a large disparity of results in the literature linked to various properties of these instabilities. The local Rossby number, Ro, is no longer constant and may vary with the radius (Gauthier et al. 1999, Serre et al. 2001) and this affects the global stability properties of the boundary layers. Thus, the stability of the flow is governed by the competition between destabilizing non-linear effects and non-parallel effects known to be stabilizing in linear theory (Othman \& Corke 2006, Davies \& Carpenter 2003).

Thus, the critical parameters of the global instability for the onset of Type I and Type II may depart from those of the Ekman and the Bödewadt boundary layers for a single disk, Serre et al. (2004). In general, boundary layers in finite-disks cavities appear to be slightly more stable than the corresponding similarity solutions. Tatro \& Mollo-Christensen (1967) found a linear dependence of the local critical Reynolds numbers on Rossby number: $\operatorname{Re}_{r c l}=124.5-7.32 \mathrm{Ro}$ and $\mathrm{Re}_{r c I}=56.3-116.8 \mathrm{Ro}$, results that have recently been confirmed by Viaud et al. (2008) using spectral DNS in a rotating-disk cavity with radial through-flow (an arrangement with many similarities to the present flow). Several authors have also mentioned that the most unstable azimuthal wavenumber $\beta_{\max }$ (i.e. the number of spiral arms) and its related frequency change with $\operatorname{Re}_{r}$ and Ro. Lingwood (1997) specified theoretically that $\beta_{\max }$ is proportional to $\mathrm{Re}_{r} / \mathrm{Ro}$. Since $\mathrm{Re}_{r}$ and $\mathrm{Ro}$ are both functions of the radius, it is difficult to compare theoretical values of $\beta_{\max }$ with experimental or numerical observations in an enclosed 
cavity. In general, the observed values of $\beta_{\max }$ are smaller than those expected from theory. The presence of roughness at the disk surface in the experiments and of inadequate mesh refinement in the numerical solutions can also greatly affect the number of spiral arms. The observation of stationary modes in experiments, which should however be less unstable than travelling ones, is also related to wall roughness as shown by several theoretical studies (Wilkinson \& Malik 1983, Mack 1985, Balachandar et al. 1992, Pier 2003). Recent investigations by different authors (Takagi \& Itoh 2004, White \& Saric 2005) have concluded that the disturbances related to such roughness are certainly stronger than any perturbations induced by the turbulent upstream flow capable of generating the unsteady modes. Such roughness could also explain the difficulty of observing the axisymmetric mode of instability in cavities since these require particularly clean disk surfaces. Nevertheless, these differences between theory and observations may also be related to incorrect predictions by the linear theory due to the omission of certain terms, such as the curvature of the streamlines. For example, Malik et al. (1981) using spatial theory and Kobayashi et al. (1980) using a temporal theory have shown that linear theories taking into account both Coriolis and streamlinecurvature effects give values of $\beta_{\max }$ in better agreement with observations.

\subsection{Instability patterns}

In the limit of very thin cavities, $G<<1$, instability patterns between Regimes I and III (Figure 2) have been considered only by Schouveiler et al. (2001) (Figures 3) and Cros \& Le Gal (2002) using, respectively, experimental flow visualizations and image processing. The single instability pattern reported before turbulence is related to negative spirals, denoted SRIII (Figures 4a) which occur at the periphery of the cavity at large radius.

As would be expected from linear stability theory for a single disk, the primary destabilization in the Batchelor regime occurs in the stator boundary-layer in the form of travelling waves 
which disappear at a local radius corresponding to the critical Reynolds number in cylindrical cavities. In an annular cavity, when the inner radius is larger than that corresponding to the critical Reynolds number, the waves persist to the hub and disturbances are transmitted to the rotor boundary-layer, eventually triggering instability. Otherwise, the influence of a central hub on the critical Reynolds number is weak since the latter is only slightly decreased when $R_{m}$ is increased (Poncet et al. 2009).

The first instability is characterized by a circular wave (CR in Figures 3, 5a) propagating in the flow direction and recognized as a Type II instability (Pikhtov \& Smirnov 1992, Serre et al. 2004). Lopez \& Weidman (1996) showed that this structure, first observed experimentally by Savas (1983) during spin-down, does not arise from the interaction of an external upstream perturbation with the stator boundary layer, but rather a response to the local structure of the boundary layer. For steady conditions in rotor-stator cavities, such vortices have been reported much later both experimentally (Gauthier et al. 1999, Schouveiler et al. 1999) (Figure 5a) and numerically using fully three-dimensional computations (Serre et al. 2001) (Figure 5b) even for annular cavities.

The most detailed study has been carried out by Gauthier et al. (1999) who have shown that this instability has all the properties of a supercritical bifurcation at $\operatorname{Re}_{r c}=75$ with the most unstable temporal frequency $\sigma=4$, in agreement with numerical solutions, Serre et al. (2001) which predict frequencies $\sigma$ in the range 1-4 for $G$ between 0.2 and 0.5 . It is also noted in this connection that Lingwood (1997) obtained theoretically the value $\sigma=1.3$ at the convective/absolute transition for axisymmetric structures in the Bödewadt layer. Above this threshold, the instability is linearly convective leading to a high sensitivity to external controlled or uncontrolled forcing (Gauthier et al. 1999). This result has recently been confirmed numerically by Poncet et al. (2009) who have shown in particular that, in the experiments, this mode is sustained by noise involving permanent circular structures. In the 
literature, these circular waves disappear at small radii corresponding to local Reynolds $\operatorname{Re}_{r}$ in the range 25-75, depending on the confinement, i.e. the local Rossby number, Ro.

For increasing rotation rate, the axisymmetry of the flow is broken and spiral waves, denoted SRI (Figure 3) develop in the stator-layer (Figure 5b, 6) with positive angles ranging from about $10^{\circ}$ to $25^{\circ}$ (Serre et al. 2001, Schouveiler et al. 2001). Experimental (Schouveiler et al. 1999 and Cros et al. 2005) and numerical results (Poncet et al. 2009) show that this is a supercritical Hopf bifurcation. In the literature, these spiral arms are observed for $\operatorname{Re}_{r c}>50$, i.e. a value slightly larger than for the Type II axisymmetric mode. At these Reynolds numbers, nearly the whole of the stationary disk boundary layer is absolutely unstable. At the lowest rotation rates, the spiral wave pattern can coexist with the previous circular waves (Figure 6a) which are noise sustained: spirals appear around the periphery and circles are observed at small radii corresponding to lower local Reynolds numbers, $\mathrm{Re}_{r}$. According to the linear stability analysis of the Batchelor flow by San'kov \& Smirnov (1991) and Serre et al. (2004), this spiral mode of positive angle is a Type I instability.

Both experimental observations and numerical solutions typically exhibit spiral patterns with an azimuthal wavenumber, $\beta$, in the range 16-30, depending on the value of the aspect ratio and rotation rate. Schouveiler et al. (1998) showed that the wavenumber selection process of this secondary instability can be regarded as resulting from an Eckhaus instability that selects the number of spiral arms. Thus, the number of arms is 18 at threshold but, depending on the time history of the flow, states with 16 to 24 arms may also be obtained.

The rotor layer is less well documented. Experimentally, the measurements are technically more difficult than those on the stator and numerically, the occurrence of instabilities at high rotation rates involves large computational costs due to resolution requirements. Only a few numerical results exist in the laminar regime. For annular cavities Serre et al. (2001) showed that the stator-layer instabilities are convected along the hub and induce disturbances in the 
rotor layer triggering first axisymmetric and later three-dimensional convective modes characterized by a negative angle $\varepsilon$ in the range $-7.5^{\circ} \leq \varepsilon \leq-20^{\circ}$ (Figure 6b). These convective modes were also numerically observed by spectral DNS by Viaud et al. (2008) in the rotor-layer of a cavity with radial through-flow, and showed similar characteristics to the Type II instability predicted by Lingwood (1997) and Serre et al. (2004) at the same Rossby numbers.

\subsection{Transition to turbulence}

The evolution to higher bifurcations in the rotor-stator cavity has received much less attention. In the limit of very thin cavities, $G<<1$ and merged boundary layers, the most interesting feature observed by Cros \& Le Gal (2002) is that transition to turbulence occurs via defect turbulence in the network of negative spirals previously denoted SRIII (Figures 3, 4a). Increasing further the rotation rate leads to the appearance of turbulent domains within a laminar background as can occur in a narrow-gap Taylor-Couette system between two concentric cylinders. These instabilities, in the form of turbulent spirals (SW, Figures 3, 4b) periodically located around the disk, may be related to a turbulent form of the steady solitary vortex solution calculated by Hoffmann \& Busse (2000) in the Ekman-Couette layer. By increasing progressively the rotation rate, turbulent spots (SP, Figures 3, 4c) appear and finally invade the whole cavity.

In the Batchelor regime, turbulence firstly occurs in the stator layer. Transition mechanisms are difficult to identify in such layers due to the speed at which turbulence ensues. To our knowledge, the experiment of Cros et al. (2005) is the only one to provide some insight into the evolution and further transitions of the stator layer for moderate Reynolds numbers, Re, up to 73890 . The transition to turbulence seems to be governed by nonlinear interactions of the circular and spiral modes of the stationary disk flow identified at 
lower rotation rates. These authors reported, at $\mathrm{Re}=32840$, only one period-doubling bifurcation before its complete destruction by transition to wave turbulence. No relation between the bifurcation and the appearance of phase defects could be determined, however. On increasing rotation rates further, a more developed turbulence issuing from the former chaotic waves was observed.

Recent numerical studies (Séverac et al. 2007, Viaud et al. 2008) provide some insight into transition in the rotor boundary layer. Interest has been stimulated by the experimental and theoretical studies of Lingwood $(1996,1997)$ which showed that the onset of absolute instability in both the von Kármán and Ekman layers adjacent to a single disk occurred at a value of Reynolds number which closely corresponded to that obtained experimentally for laminar-turbulent transition. This major contribution to the turbulent breakdown process opened the possibility of a direct route towards turbulence. Further related investigations have been made by Pier (2003), Davies \& Carpenter (2003), and Viaud et al. (2008). Even if this characterization in terms of convective and absolute instability is not rigorously applicable in the context of confined flows, it can bring helpful insight to the breakdown to turbulence in a rotor-stator cavity (Serre et al. 2004). At high local Reynolds numbers ( $\left.\operatorname{Re}_{r}>400\right)$, the rotorlayer becomes absolutely unstable and the Type I instability with spiral arms of positive inclination are observed. High-order LES from Séverac et al. (2007) confirm the presence of spiral arms in the rotor layer at $\operatorname{Re}_{r} \approx 400$ with all the characteristics of the absolute Type I instability and, just upstream, a turbulent region located at a radial position very close that predicted by Lingwood's theory assuming parallel flow. Further theoretical investigation by Pier (2003) showed that this saturated Type I absolute mode could be overtaken by a direct route to turbulence through a secondary absolute instability. Nevertheless, the question of the transition scenario in the rotor-layer remains open. 


\section{TURBULENT FLOW}

\subsection{Statistically-Steady, Axisymmetric View}

Until relatively recently, the turbulent flow regime has been treated, both in experiments and computations, as though the flow pattern within the disk cavity were axisymmetric. Experiments have employed pitot tubes, hot-wire anemometers and LDA to obtain circumferential and radial mean velocity profiles and (in the case of hot-wire and LDA studies) rms turbulence traverses across the cavities at selected radii. The relevant literature up to the late 1980's has been comprehensively reviewed by Owen \& Rogers (1989). Since then, detailed studies for closed cavities without an inner hub have been reported, among others, by Itoh et al. (1992) for $G=0.08$, Cheah et al. (1994) for $G=0.127$ and, most recently, by Poncet et al. (2005a, b) for a narrow annular cavity, $G=0.036$, as the base case of a study mainly concerned with the effects of radial through-flow. Representative profiles from Itoh et al. (1992) are shown in Figure 7.

The first major experimental and theoretical study of turbulent flows in rotor-stator systems was carried out by Daily \& Nece (1960) who identified the four flow regimes shown in Figure 2. The most investigated regime has been Regime IV where the two boundary layers are separated by a non-viscous core region. Using the Taylor-Proudman theorem, the entrainment coefficient $K$ can readily be linked to the radial pressure gradient at the rotor which is crucial to the design of thrust bearings. Poncet et al. (2005a) obtained $K=0.438$ by direct velocity measurements, which coincides with the asymptotic value given by their analytical model. $K$ appears to be independent of the inter-disk spacing $h$ but very sensitive to the core-swirl ratio at the periphery of the cavity. For a comparison with available values of $K$ in shrouded or un-shrouded systems see Randriamampianina et al. (1997) and Poncet et al. (2008). 
Clearly, the boundary-layer thickness on the spinning disk is appreciably less than on the stator as evidenced by radial and tangential mean velocities, Figure 7, and the turbulence intensities. The difference was attributed by Itoh et al. (1992) to a greater stability of the radially outward flow in the Ekman layer than the return flow in the Bödewadt layer. Cheah et al. (1994), however, suggested the cause was due principally to convection, i.e. to the fact that fluid in the Bödewadt layer is essentially downstream from the Ekman layer. At the Reynolds numbers of these investigations, there is an appreciable region near the axis where the Ekman boundary layer is effectively viscous. Predicting the extent of that region over the range of geometric and flow parameters of interest has proved a challenging task for computational modelling.

Throughout the 1980's flow modelling within the cavity was based on axisymmetric RANS computations with some form of isotropic eddy-viscosity model. The early studies of Chew (1985) employed the form of mixing-length hypothesis used earlier by Koosinlin et al. (1974) for flow on spinning free disks. The first application of a two-equation model to a rotating disk system appears to have been that of Gosman et al. (1976). Like Chew (1984) some years later, they used the two-equation $k-\varepsilon$ model. These first attempts were relatively unsuccessful, however, because of the use of simple log-law wall functions to bridge the viscosity-affected sublayers next to the disks. In fact, the velocity vector parallel to the disks undergoes strong skewing across the sublayer so it is hardly surprising that any formula assuming the direction of the velocity in the fully turbulent near-wall region to be the same as the wall-shear-stress vector should exhibit serious prediction errors. Later, in place of wall functions, Iacovides \& Theofanopoulos (1991) blended a two-equation modelling of the fully turbulent region with the mixing-length hypothesis across the sublayers. A more elaborate modelling was adopted by Morse (1988) who adopted the Launder-Sharma (1974) "lowReynolds-number" form of the $k-\varepsilon$ model over the complete cavity. His results displayed 
significant improvements over mixing-length schemes though, to avoid a tendency for the boundary layer on the rotor to display a too large laminar-like region, he later (Morse 1991) partly replaced, in the sublayer "damping" functions, the local turbulence Reynolds number, $k^{2} / v \mathcal{E}$, by the conventional normalized wall distance, $y^{+}$. This route was subsequently adopted by several other workers of the period though, as Elena (1994) remarked, the introduction of the wall-normal distance was "prejudicial to the generality of application".

By the 1990s it was acknowledged that for more reliable predictions one should abandon linear eddy-viscosity schemes. A first step by Iacovides \& Theofanopoulos (1991) was the modelling of the stress field by an ASM (effectively, a non-linear eddy viscosity model) though this was applied only in the fully turbulent region with the mixing-length hypothesis being retained across the sublayers. Later, however, Iacovides \& Toumpanakis (1993) moved to a full second-moment closure (solving transport equations for all six Reynolds stresses) including the sublayers. Closure at this level represented an important conceptual advance because then the direct effects of rotation on the individual Reynolds stresses were directly accounted for (whereas there is no Coriolis force on the turbulence energy). Perhaps the most valuable discovery to emerge from the Iacovides \& Toumpanakis (1993) work, however, was that corrections to the transport equation for $\varepsilon$ (developed in other work to dampen the excessive length scales in separated flows) also led to marked improvements in disk-cavity flows.

Elena \& Schiestel (1993) also adopted second-moment closure for their first rotor-stator studies but concluded that the wall-proximity corrections employed in their model, (developed much earlier for external boundary layers on plane surfaces), were unsuitable for internal flows. It was timely, therefore, that a new type of second-moment closure had by then begun to be used in complex flows - one that satisfied the two-component limit (TCL), Lumley (1978), a state that is asymptotically reached as one approaches a wall.. The 
application of the TCL constraint to modelling mean-strain effects on the pressure-strain correlation proved crucial (Launder \& Li 1994) because then pressure-reflection impacts from the wall were, to a large extent, embedded in the basic model rather than having to be added as an empirical correction. The first application of a prototype form of the model to rotating flows by Launder \& Tselepidakis (1994) led Elena \& Schiestel (1996) (see also Elena 1994) to adopt and further refine the model for a range of disk-cavity studies. Figure $\mathbf{8}$ compares their prediction of the closed cavity of Itoh et al. (1992) with the measured behaviour: agreement with the data is markedly better than with their earlier model (Elena \& Schiestel 1993). Very satisfactory computed behaviour was also reported by Elena (1994) for the wider cavity of Cheah et al (1994). Recently Haddadi \& Poncet (2008), from the same research group, have applied the model to the very narrow disk cavity of Poncet et al. (2005b), $G=0.036$, including cases with radial through-flow.

\subsection{Three- Dimensional, Time-dependent View}

The treatment of the turbulent flow regime as statistically stationary implicitly assumed that the coherent structures present in the laminar and transitional modes either disappeared or were "drowned out" by intense fine-scale turbulence. Owen (2000) first suggested that, sometimes, the flow within the disk cavity might not be steady. Summarizing research at Bath University on cavities with through-flow, he noted that their usual RANS model failed to converge for certain ranges of parameters and speculated that some form of organized largescale structure may have been present in the actual flow.

The first computations of the rotor-stator disk cavity which treated the flow as unsteady were the DNS explorations of Lygren \& Andersson (2001). To achieve a sufficiently fine mesh to resolve the smallest eddies, only a sector of the flow was covered, with boundary information being transferred from one side of the sector to the other. The conditions 
approximated one of the tests by Itoh (1995) with $G=0.08$. Agreement with the experimental (circumferentially averaged) velocity profiles was close but no spiral vortices, analogous to those reported by Serre et al. (2001) in laminar/transitional flows, were found in the near-wall boundary layers. Subsequently, Czarny et al. (2002) carried out simple flow-visualization experiments in a rotating water table for empty rotor-stator disk-cavities. These displayed traces of the near-disk spirals, but also quite unexpected large-scale vortices in the core region where the mean flow rotates at roughly half the speed of the rotor, Figure 9. Moreover, the pattern revealed by the dye trace showed 5, 3 or 2 eddies - or even none - depending upon the aspect ratio of the cavity and the rotor speed! Clips of the rotating flow structures may be viewed on the following Manchester University website: http://tmgflows.mace.manchester.ac.uk/index.html.

Wu \& Squires (2000) were the first to apply LES to the statistically three-dimensional turbulent boundary layer over a single rotating disk. Their results supported the observations of Littell \& Eaton (1994) regarding the mechanisms promoting sweeps and ejections which play a role in shear-stress production. Andersson \& Lygren (2006) performed wide-gap and narrow-gap simulations to investigate the degree of three-dimensionality in both Ekman and Bödewadt layers for $R e \leq 1.6 \times 10^{6}$. Their results also support the conclusion of Littell \& Eaton (1994), that the mean flow three-dimensionality affects the near-wall vortices and their ability to generate shear stresses. The first LES simulation in an enclosed rotor-stator cavity was reported by Séverac et al. (2007) using a spectral-vanishing-viscosity approach up to $\operatorname{Re}=10^{6}$. Their results compare very favourably with LDV measurements in a cavity of the same proportions and highlight more specifically that the three-dimensionality of both boundary layers is much greater than in the idealized system studied by Andersson \& Lygren (2006). While, as seen in Figure 10, coherent near-wall vortices were accurately reproduced by these simulations, Séverac et al. (2007) did not find the large-scale core vortices that were 
plainly evident in the experiments. Recently, the probable reason for this failure has emerged. Rather than mapping a complete empty cavity, which would have posed major computational problems (due to the excessive concentration of nodes that the spectral scheme inevitably produced at the axis), the computations adopted an annular configuration with the inner cylinder radius $\approx 30 \%$ of that of the outer disk and frictionless. However, in a recent unpublished extension of the experiments of Czarny et al. (2002), the present authors have found that when the circular disk was converted to an annulus by the insertion of a hub cylinder to match the configuration of Séverac et al (2007), the organized structures shown in Figure 9 for the empty cavity were absent. It appears that the inclusion of the inner cylinder blocks the transfer via the pressure field of crucial information essential to the creation of the large-scale structures.

Meanwhile, Craft et al. (2008) have reported three dimensional URANS computations in the search for organized rotating structures. The first results, using a very fine near-wall grid to resolve accurately the low-Reynolds-number $k$ - $\varepsilon$ model, found Ekman and Bödewadt spirals but, again, no organized large-scale structures such as those reported by Czarny et al. (2002). Subsequently, however, while retaining the $k$ - $\varepsilon$ model in the core of the flow, they adopted a new analytical wall function to bridge the semi-viscous wall layer that permitted skewing of the wall-parallel velocity vector across the sublayer. This development allowed a much greater density of grid points over the remainder of the cavity. The results from these computations (Zacharos 2009, Craft et al. 2008) not only reproduced the near-wall spirals, Figure 11a, but also large-scale two- or three-lobed structures in the core of the flow, Figure 11b,c. However, agreement with the observed behaviour is less than it might appear; for the structures exhibit a progressive evolution in time, the three-lobed version collapsing to two lobes and, thereafter, decaying to what, at the end of the computed time evolution, appears to be a flow heading to axisymmetric form. By contrast the structures visualized by Czarny et al. 
(2002) were assuredly permanent. Zacharos (2009) explored whether the introduction of a second-moment closure in the core (in place of the eddy viscosity model) might alter the picture. It did, only then no organized structures were formed even in the initial phase. Clearly, the issue of resolving fully the observed flow structure in a rotating disk cavity has some way still to run!

\section{ACKNOWLEDGMENTS}

We acknowledge with appreciation the indirect contributions of Patrick Bontoux, MariePierre Chauve, Hector Iacovides, Patrice Le Gal and Roland Schiestel to this review. Authors' names are listed in alphabetical order. 


\section{LITERATURE CITED}

Abrahamson SM, Eaton JK, Koga DJ. 1991. The flow between shrouded co-rotating disks. Phys. Fluids A 1: 241-51

Andersson HI, Lygren M. 2006. LES of open rotor-stator flow. Int. J. Heat Fluid Flow, 27(4): $551-57$

Balachandar S, Street CL, Malik MR. 1992. Secondary instability in rotating-disk flow. J. Fluid Mech. 242: 323-47

Batchelor GK. 1951. Note on a class of solutions of the Navier-Stokes equations representing steady rotationally-symmetric flow. Quart. J. Mech. and Appl. Math. 4 (1): 29-41

Bödewadt UT. 1940. Die Drehströmung über festem Grunde. Z. Angew. Math. Mech. 20: 24153

Brady JF, Durlofsky L. 1987. On rotating disk flow. J. Fluid Mech. 175: 363-94

Cheah SC, Iacovides H, Jackson DC, Ji H, Launder BE. 1994. Experimental investigation of enclosed rotor-stator disc flows. Experimental Thermal \& Fluid Science 9: 445-55

Chew JW. 1984. Prediction of flow in rotating disc systems using the $k$ - $\varepsilon$ turbulence model. ASME Gas Turbine Conference, Amsterdam, Paper ASME 84-GT-229

Chew JW. 1985. Prediction of flow in rotating cavity with radial outflow using a mixinglength turbulence model. Proc. $4^{\text {th }}$ Int. Conf. on Num. Methods in Laminar \& Turbulent Flow, pp. 318- 329. Swansea: Pineridge Press

Cooper P, Reshotko E. 1975. Turbulent flow between a rotating disk and a parallel wall. AIAA J. 13 (5): 573-78

Craft TJ, Iacovides H, Launder BE, Zacharos A. 2008. Some swirling-flow challenges for turbulent CFD. Flow, Turbulence \& Combustion 80: 419-34

Crespo del Arco E, Serre E, Bontoux P, Launder BE. 2005. Stability transition and turbulence in rotating cavities, Instability of Flows, ed. M Rahman, Southampton: WIT Press.141-95 
Cros A, Le Gal P. 2002. Spatiotemporal intermittency in the torsional Couette flow between a rotating and a stationary disk. Phys. Fluids 14 (11): 3755-65

Cros A, Floriani E, Le Gal P, Lima R. 2005. Transition to turbulence of the Batchelor flow in a rotor/stator device. Eur.J. Mech.B/Fluids 24: 409-24

Czarny O, Iacovides H, Launder BE. 2002. Precessing vortex structures in turbulent flow within rotor-stator disc cavities. Flow, Turbulence and Combustion 69: 51-61

Daily JW, Ernst WD, Asbedian VV. 1964. Enclosed rotating disks with superposed throughflow: mean steady and periodic unsteady characteristics of induced flow. Report 59901004, Department of Civil Engineering, Massachusetts Institute of Technology

Daily JW, Nece RE. 1960. Chamber dimension effects on induced flow and frictional resistance of enclosed rotating disks. ASME J. Basic Eng. 82: 217-32

Davies C, Carpenter PW. 2003. Global behaviour corresponding to the absolute instability of the rotating-disk boundary layer. J. Fluid Mech. 486: 287-329

Dyment A. 1981. Formulation asymptotique des écoulements d'un fluide incompressible entre deux disques coaxiaux voisins en rotation. C. R. Acad. Sci. II 292 : 129-32

Ekman VW. 1902. Om Jordrotationens inverkan på vindstrømmar I hafvet. Nytt Mag. Naturvid. 40: 37-62

Elena L. 1994. Modélisation de la turbulence inhomogène en présence de rotation. $\mathrm{PhD}$ thesis. Université d'Aix-Marseille II, 227 pp.

Elena L, Schiestel R. 1993. Numerical prediction of the turbulent flow in rotor stator systems. Engineering Turbulence Modeling \& Measurement-2, Ed. W Rodi \& F Martelli, pp.765-74.

Elena L, Schiestel R. 1996. Turbulence modeling of rotating confined flows. Int. J. Heat \& Fluid Flow 17: 283-89

Escudier MP. 1984. Observations of the flow produced in a cylindrical container by a rotating end wall. Exp. Fluids. 2: 187-96 
Faller AJ. 1991. Instability and transition of disturbed flow over a rotating disk. J. Fluid Mech. 230: 245-69

Gauthier G, Gondret P, Rabaud M. 1999. Axisymmetric propagating vortices in the flow between a rotating and a stationary disk enclosed by a cylinder. J. Fluid Mech. 386: 105-26 Gosman AD, Lockwood FC, Loughhead JN. 1976. Prediction of recirculating, swirling flow in rotating disc systems. J. Mech. Eng. Sci. 18: 142-48

Haddadi S, Poncet S. 2008. Turbulence modelling of torsiional Couette flows. Int. J. Rotating Machinery, Article ID 635138

Hoffmann NP, Busse FH, Chen WL. 1998. Transitions to complex flows in the EkmanCouette layer. J. Fluid Mech. 366: 311-31

Humphrey JAC, Schuler CA, Webster DR. 1995. Unsteady laminar flow between a pair of discs co-rotating in a fixed cylindrical container. Phys. Fluids A 7: 1225-40

Iacovides H, Theofanopoulos IP. 1991. Turbulence modeling of axisymmetric flow inside rotating cavities. Int. J. Heat \& Fluid Flow 12: 2-11

Iacovides H, Toumpanakis P. 1993. Turbulence modeling of flows in axisymmetric rotorstator systems. Proc. $5^{\text {th }}$ Int Symp. on Refined Flow Modelling \& Turbulence Measurements, Int. Ass'n Hydr. Res., Paris: 835-42

Itoh M. 1991. On the instability of flow between coaxial rotating disks. ASME Boundary Layer Stability and Transition to Turbulence FED 114: 83-89

Itoh M, Yamada Y, Imao S, Gonda M. 1992. Experiments on turbulent flow due to an enclosed rotating disk. Exp. Thermal \& Fluid Sci. 5: 359-68

Itoh M. 1995. Experiments on the turbulent flow in the narrow clearance between a rotating and a stationary disk. In Turbulent Flows, ed. BF Carroll, T Kobayashi, MJ Morris, ASMEFED 208 : 27-32 
Itoh M, Yamada Y, Imao S, Gonda M. 1992. Experiments on turbulent flow due to an enclosed rotating disc. Exp. Thermal Fluid Sci. 5: 359-68

Kobayashi R, Kohama Y, Takamadate C. 1980. Spiral vortices in boundary layer transition regime on a rotating disk. Acta Mechanica 35: 71-82

Koosinlin ML, Launder BE, Sharma BI. 1974. Prediction of momentum heat and mass transfer in swirling turbulent boundary layers. ASME J. Heat Transfer 96: 204-09

Kreiss HO, Parter SV. 1983. On the swirling flow between rotating coaxial disks: existence and uniqueness. Commun. Pure Appl. Math. 36: 55-84

Launder BE, Li SP. 1994. On the elimination of wall topography parameters from secondmoment closure. Phys. Fluids 6: 999-1006

Launder BE, Sharma BI. 1974. Application of the energy-dissipation model of turbulence to the calculation of flow near a spinning disk. Letters in Heat Mass Trans. 1: 131-38

Launder BE, Tselepidakis DP. 1994. Application of a new second-moment closure to turbulent channel flow rotating in orthogonal mode. Int. J. Heat \& Fluid Flow 15: 2-10

Lilly DK. 1966. On the instability of Ekman boundary layer. J. Atm. Sci. 23: 481-94

Lingwood RJ. 1996. An experimental study of absolute instability of the rotating-disk boundary-layer flow. J. Fluid Mech. 314: 373-405

Lingwood RJ. 1997. Absolute instability of the Ekman layer and related rotating flows. $J$. Fluid Mech. 331: 405-28

Littell HS, Eaton JK. 1994. Turbulence characteristics of the boundary layer on a rotating disk. J. Fluid. Mech., 266:175-207

Lopez JM, Weidman PD. 1996. Stability of stationary endwall boundary layers during spindown. J. Fluid Mech. 326: 373-98

Lumley JL. 1978. Computational modeling of turbulent flows. Adv. Appl. Mech. 18: 123-76 
Lygren M, Andersson HI. 2001. Turbulent flow between a rotating and a stationary disk. $J$. Fluid. Mech. 426: 297-326

Mack LM 1985. The wave pattern produced by point source on a rotating disk. AIAA Paper 85-0490

Malik MR, Wilkinson SP, Orszag SA. 1981. Instability and transition in rotating-disk flow. AIAA J. 19 (9): 1131-38

Moisy F, Doaré O, Pasutto T, Daube O, Rabaud M. 2004. Experimental and numerical study of the shear layer instability between two counter-rotating disks. J. Fluid. Mech., 504:175-202 Morse AP. 1988. Numerical prediction of turbulent flow in rotating cavities. ASME J. Turbomachinery 110: 202-12

Morse AP. 1991. Application of a low-Reynolds-number k- $\varepsilon$ model to high speed rotating cavity flows. ASME J. Turbomachinery 113: 98-105

Othman H, Corke T. 2006. Experimental investigation of absolute instability of a rotatingdisk boundary layer. J. Fluid Mech. 565: 63-94

Owen JM. 2000. Flow and heat transfer in rotating disc systems. 2000. CHTO1 Turbulence heat and mass transfer -3. ed. Y Nagano, K Hanjalic, T Tsuji , 33-58, Tokyo: Aichi Shuppan Owen JM, Rogers RH. 1989. Flow and Heat Transfer in Rotating-Disc Systems : Volume 1 Rotor-Stator Systems. Ed. WD Morris. New York: Wiley \& Sons Inc.

Pearson CE. 1965. Numerical solutions for the time-dependent viscous flow between two rotating coaxial disks. J. Fluid Mech. 21 (4): 623-33

Pier B. 2003. Finite amplitude crossflow vortices, secondary instability and transition in the rotating disk boundary layer. J. Fluid Mech. 487: 315-43

Pikhtov SV, Smirnov EM. 1992. Boundary layer stability on a rotating disk with corotation of the surrounding fluid. Izv. Ross. Akad. Nauk. Mekh. Zhidk. Gaza 5: 69-77 
Poncet S, Chauve MP, Le Gal P. 2005a. Turbulent Rotating Disk Flow with Inward Throughflow. J. Fluid Mech. 522: 253-62

Poncet S, Chauve MP, Le Gal P. 2008. Lois analytiques pour les écoulements en cavité rotorstator. Mécanique \& Industries 9(3): 227-36

Poncet S, Chauve MP, Schiestel R. 2005b. Batchelor versus Stewartson flow structures in a rotor-stator cavity with throughflow. Phys. Fluids 17: 075110

Poncet S, Serre E, Le Gal P. 2009. Revisiting the two first instabilities of the flow in an annular rotor-stator cavity. Submitted to Phys. Fluids

Randriamampianina A, Elena L, Fontaine J, Schiestel R. 1997. Numerical prediction of laminar, transitional and turbulent flows in shrouded rotor-stator systems. Phys. Fluids 9 (6): $1696-1713$

Rasmussen H. 1971. High Reynolds number flow between two infinite rotating disks. J. Aust. Math. Soc. 12: 483-501

Reed HL, Saric WS. 1989. Stability of three-dimensional boundary layers. Ann. Rev. Fluid. Mech. 21: 235-84

San'kov PI, Smirnov EM. 1991. Stability of viscous flow between rotating and stationary disks. Mekhanika Zhidkoshi i gaza 6: 79-87

Savas O. 1983. Circular waves on a stationary disk in rotating flow. Phys. Fluids 26: 3445-48

Schouveiler L, Le Gal P, Chauve MP. 1998. Stability of a traveling roll system in a rotating disk flow. Phys. Fluids 10 (11): 2695-97

Schouveiler L, Le Gal P, Chauve MP. 2001. Instabilities of the flow between a rotating and a stationary disk. J. Fluid Mech. 443: 329-50

Schouveiler L, Le Gal P, Chauve MP, Takeda Y. 1999. Spiral and circular waves in the flow between a rotating and a stationary disk. Exp. Fluids 26: 179-87 
Serre E, Bontoux P. 2001 Three-dimensional swirling flow with a precessing vortex breakdown in a rotor-stator cylinder. Phys. Fluids 13 (11): 3500-03.

Serre E, Crespo del Arco E, Bontoux P. 2001. Annular and spiral patterns between a rotating and a stationary disk. J. Fluid Mech. 434: 65-100

Serre E, Tuliska-Sznitko E, Bontoux P. 2004. Coupled numerical and theoretical study of the flow transition between a rotating and a stationary disk. Phys. Fluids 16(3): 688-706

Séverac E, Poncet S, Serre E, Chauve MP. 2007. Large Eddy Simulation and measurements of turbulent enclosed rotor-stator flows. Phys. Fluids 19: 085113

Stewartson K. 1953. On the flow between two rotating coaxial disks. Proc. Camb. Phil. Soc. 49: $333-41$

Takagi S, Itoh N. 2004. The Mode-Selection Mechanism of Cross-Flow Instability in ThreeDimensional Boundary Layers. JAXA Research and Development Report: JAXA-RR-03-022E, ISSN 1349-1148, Aerodynamics Research Group, Institute of space technology and Aeronautics, Teikyo University, Tokyo

Tatro PR, Mollo-Christensen EL. 1967. Experiments on Ekman layer instability. J. Fluid Mech. 28 (3): 531-43

Viaud B, Serre E, Chomaz JM. 2008. The elephant mode between two rotating disks. J. Fluid Mech. 598: 451-64

White EB, Saric WS. 2005. Secondary instability of crossflow vortices. J. Fluid Mech. 525: 275-308

Wilkinson SP, Malik MR. 1983. Stability experiments in rotating-disk flow. AIAA Paper 831760

Wu X, Squires KD. 2000. Prediction and investigation of the turbulent flow over a rotating disk. J. Fluid. Mech. 418: 231-264. 
Zacharos A. 2009. The use of unsteady RANS in the computation of 3-dimensional flows in rotating cavities. PhD Thesis. School of Mechanical, Aerospace \& Civil Engineering, Univ. Manchester. $471 \mathrm{pp}$.

Zandbergen PJ, Dijkstra D. 1987. Von Kármán swirling flows. Ann. Rev. Fluid Mech. 19: 46591 
Figure1: Schematic diagram of an annular rotor-stator cavity enclosed by an inner hub and an external shroud.

Figure 2: Map of the four flow regimes proposed by Daily \& Nece (1960). Merged boundary layers: I (laminar) and III (turbulent). Unmerged boundary layers: II (laminar) and IV (turbulent).

Figure 3: Transition diagram by Schouveiler et al. (2001). Curves A and B (broken lines) separate the mixed base flow $\left(\mathrm{BF}_{\mathrm{M}}\right)$ from the basic flows with separate boundary layers $\left(\mathrm{BF}_{\mathrm{S}}\right)$ and with 'joined' boundary layers $\left(\mathrm{BF}_{\mathrm{J}}\right)$, respectively. Curves 1 indicate the thresholds for circular rolls $(\mathrm{CR})$. Curves 2 , $1^{\prime}, 1^{\prime \prime}$, indicate the thresholds for spiral rolls (SRI), (SRII) and (SRIII), respectively. Curves 2" and 3" indicate the thresholds for solitary waves (SW) and turbulent spots (SP), respectively. Curve 4" is the threshold for the simultaneous disappearance of the spiral rolls (SRIII) and the solitary waves (SW).

Figure 4: Instability patterns observed in torsional Couette flows: (a) SRIII patterns, (b) turbulent spirals and (c) turbulent spots (Cros \& Le Gal 2002). The disk rotates clockwise.

Figure 5: Circular waves of Type II instability in the stator boundary-layer: (a) Experimental visualization of Gauthier et al. (1999) and (b) DNS results of Serre et al. (2004).

Figure 6: Three-dimensional modes of instability: (a) coexistence of the CR and SRI patterns in the stator boundary-layer by Gauthier et al. (1999); 18 spiral arms obtained by DNS in an annular cavity by Serre et al. (2001).

Figure 7: Mean velocity profiles in turbulent flow for four radii (From Itoh et al. 1992). $S \equiv h ; \omega \equiv \Omega$; (a) circumferential velocity; (b) radial velocity.

Figure 8: Stress-transport computations of Itoh cavity (from Elena \& Schiestel 1996): (a) radial mean velocity (b) tangential mean velocity.

Figure 9: Flow visualization of vortex structure in cavity core (from Czarny et al. 2002). Lower disk rotates clockwise, upper disc (stator) transparent. Photos show ink trace from injection at mid-height in cavity for $G=0.195$ : (a) $\operatorname{Re}=1.74 \times 10^{5}$; (b) $\operatorname{Re}=1.60 \times 10^{5}$. 
Figure 10: Flow structures in the rotor boundary layer; LES by Séverac et al. (2007) for $G=0.143$ : (a) $\operatorname{Re}=10^{5} ;(b) \operatorname{Re}=4 \times 10^{5} ;$ (c) $\operatorname{Re}=10^{6}$. Disk rotates counter-clockwise.

Figure 11: Unsteady RANS computations of flow near stator in rotor-stator cavities (from Craft. et al 2008) (a) $G=0.08 ; \operatorname{Re}=10^{6} ;$ (b) $G=0.195 ; \operatorname{Re}=0.9 \times 10^{6} ; 20$ revolutions from start; (c) $G=0.195$; $\mathrm{Re}=0.9 \times 10^{6} ; 50$ revolutions from start. 


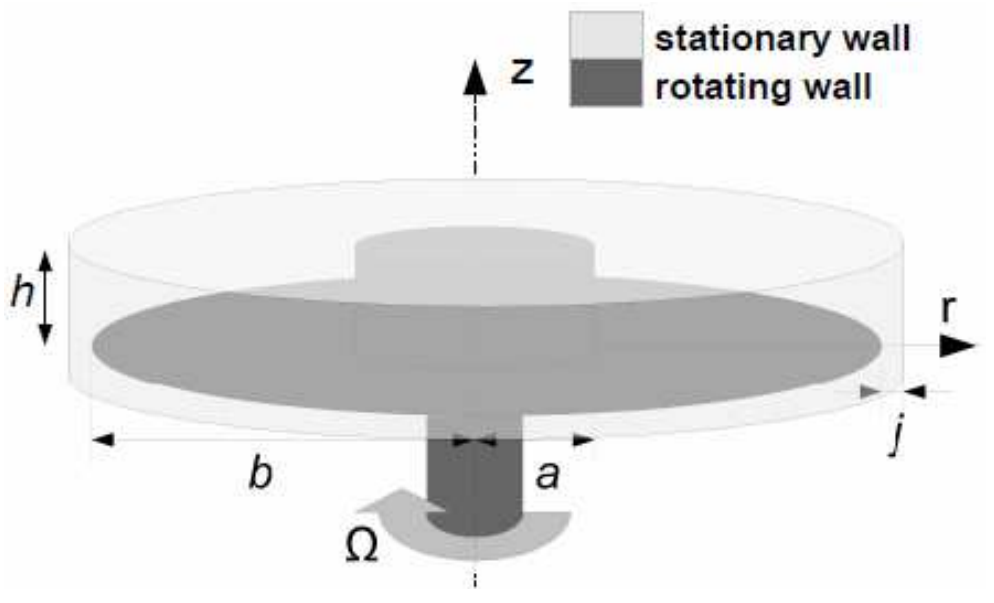

Figure1: Launder et al., Annual Rev. Fluid Mech.

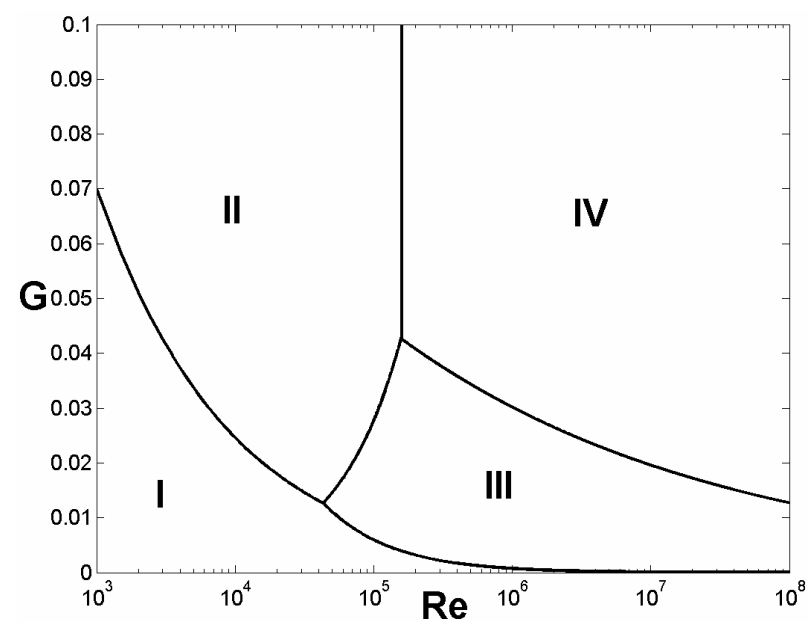

Figure 2: Launder et al., Annual Rev. Fluid Mech. 


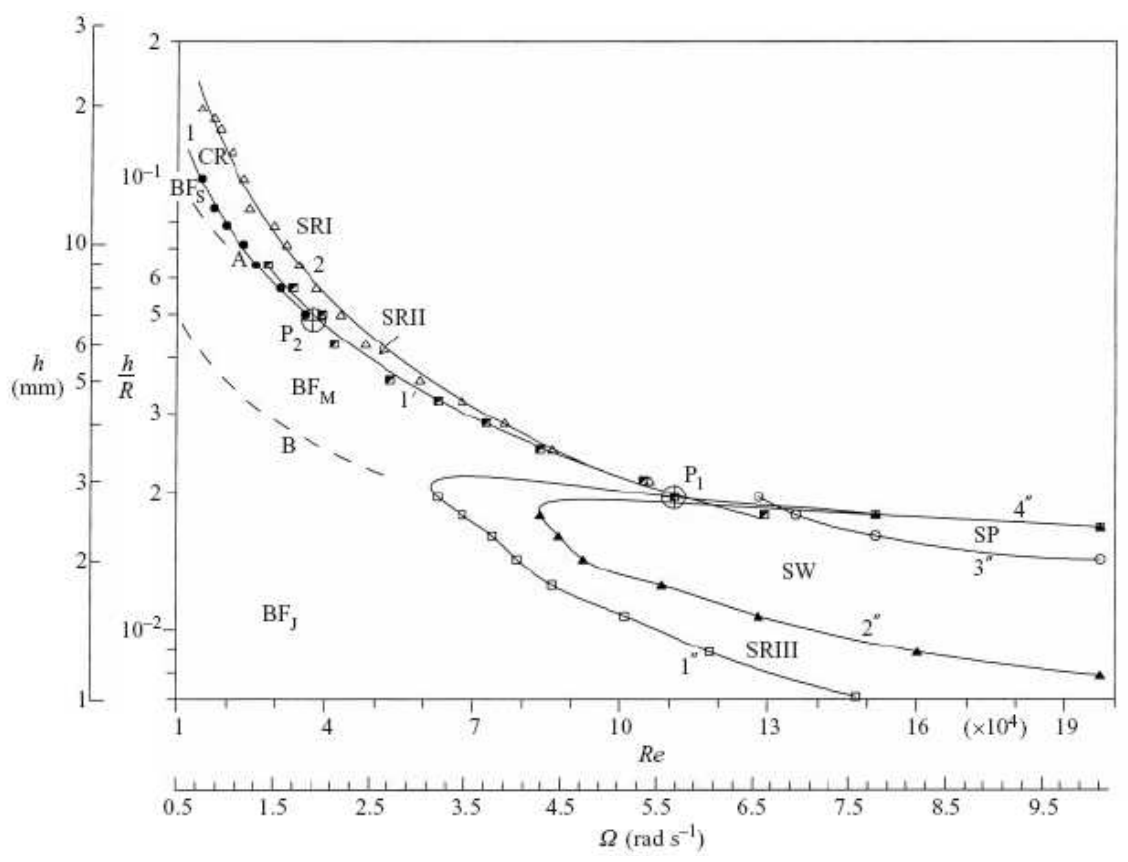

Figure 3: Launder et al., Annual Rev. Fluid Mech.

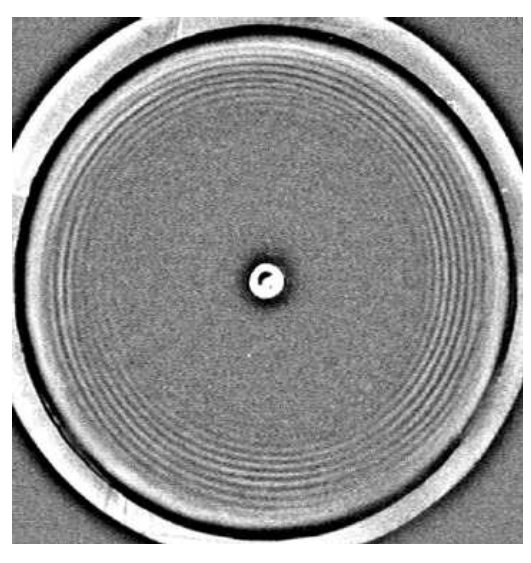

(a)

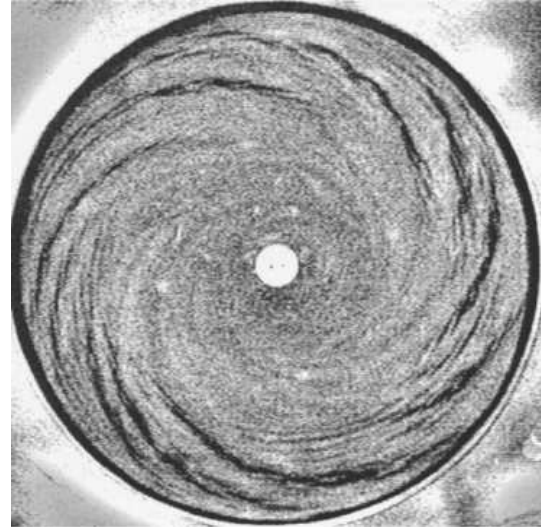

(b)

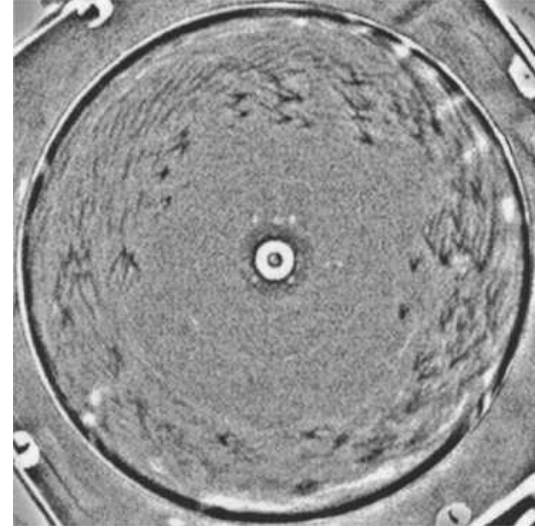

(c)

Figure 4: Launder et al., Annual Rev. Fluid Mech. 


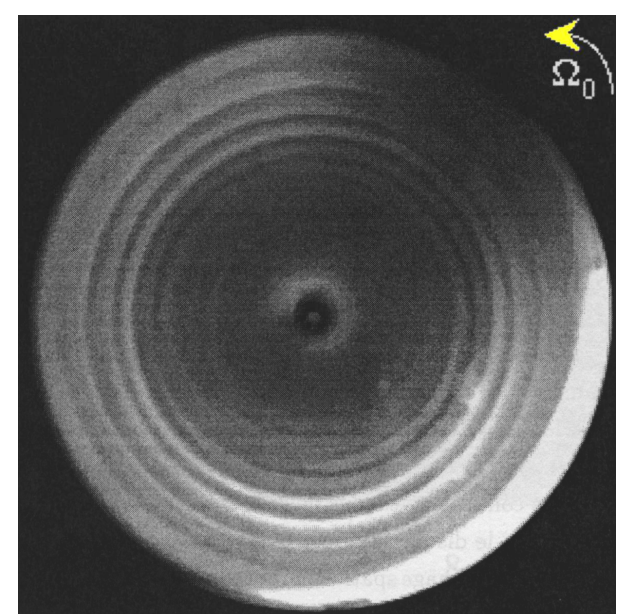

(a)

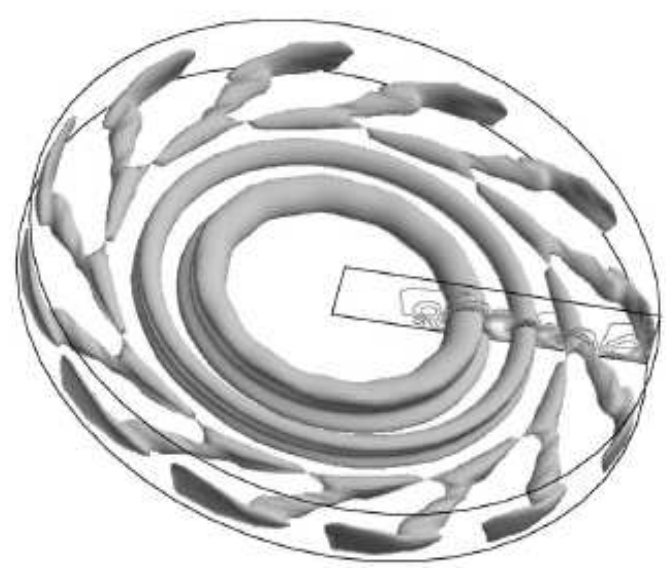

(b)

Figure 5: Launder et al., Annual Rev. Fluid Mech.

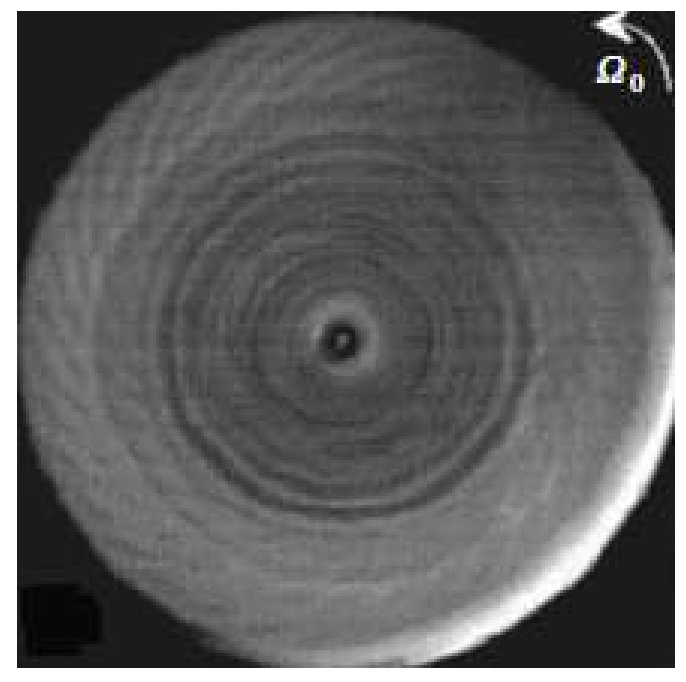

(a)

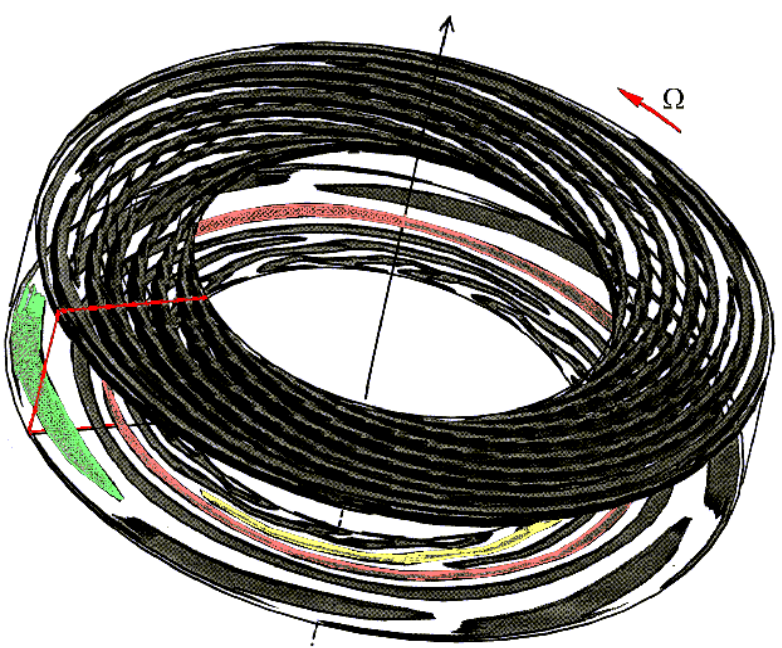

(b)

Figure 6: Launder et al., Annual Rev. Fluid Mech. 


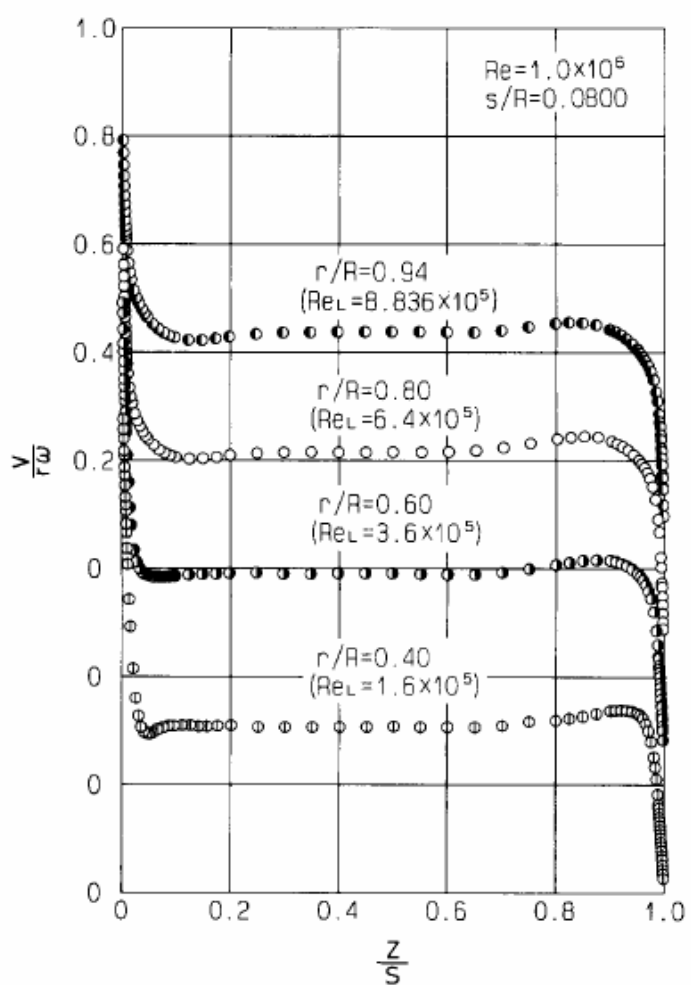

(a)

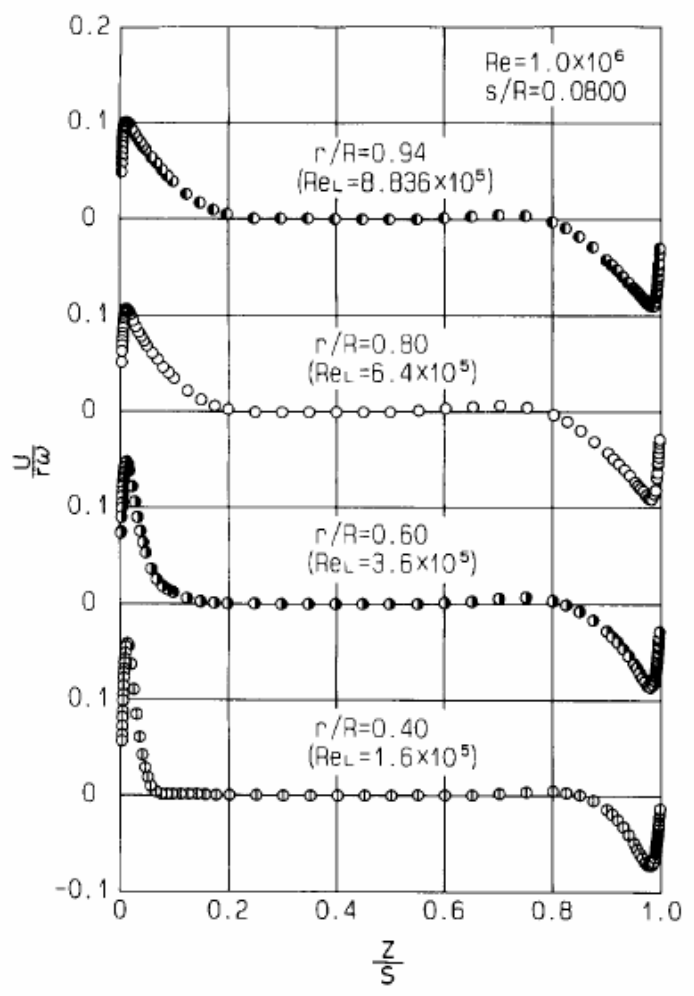

(b)

Figure 7: Launder et al., Annual Rev. Fluid Mech.

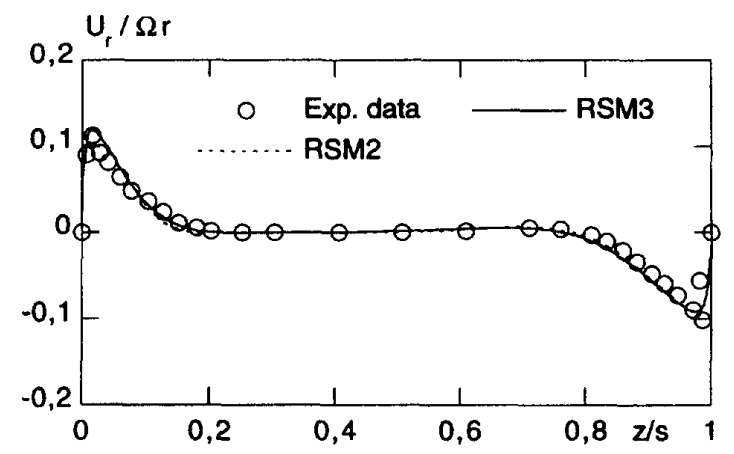

(a)

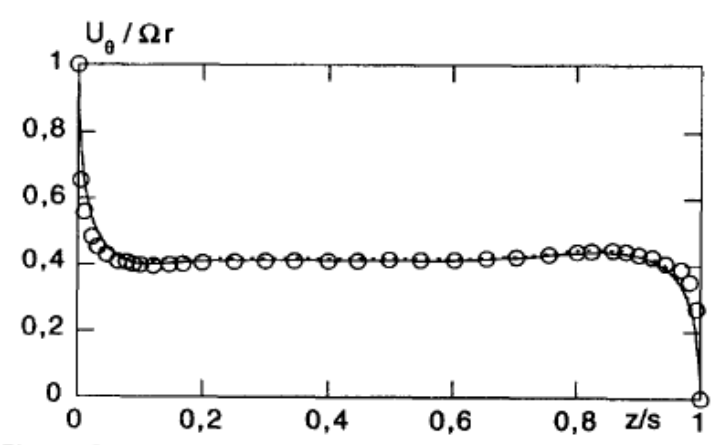

(b)

Figure 8: Launder et al., Annual Rev. Fluid Mech. 


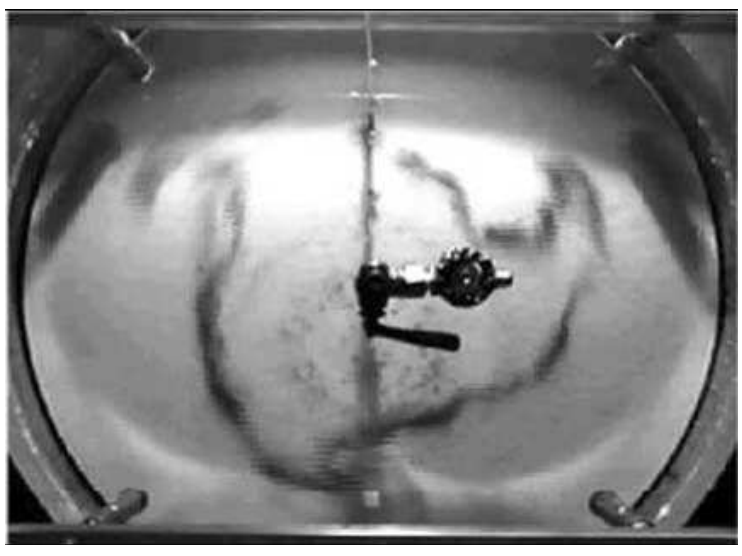

(a)

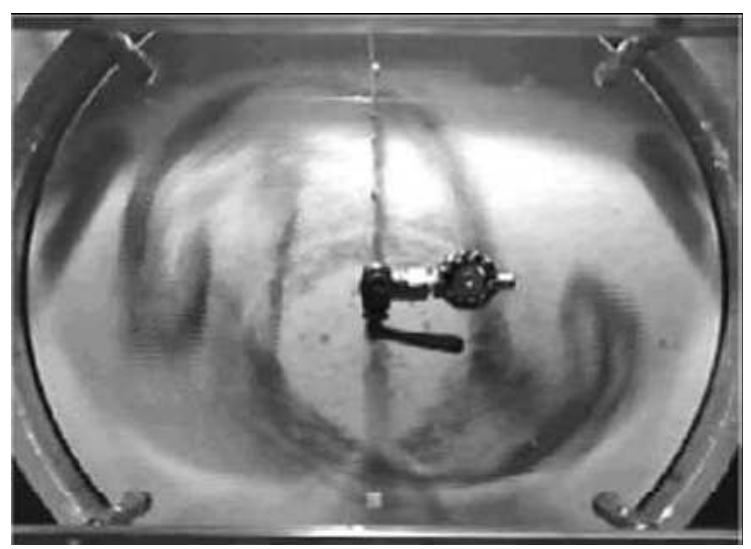

(b)

Figure 9: Launder et al., Annual Rev. Fluid Mech.

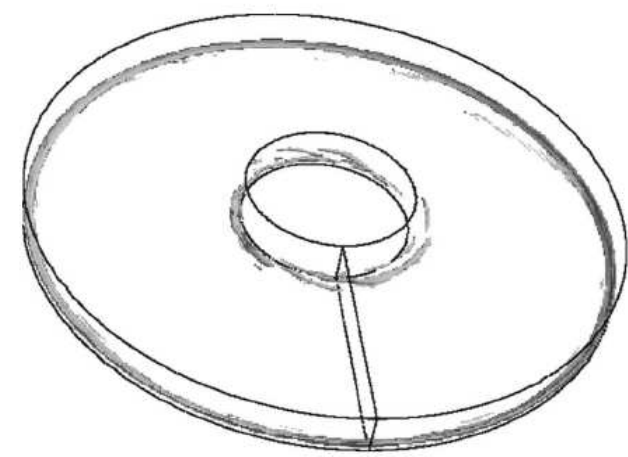

(a)

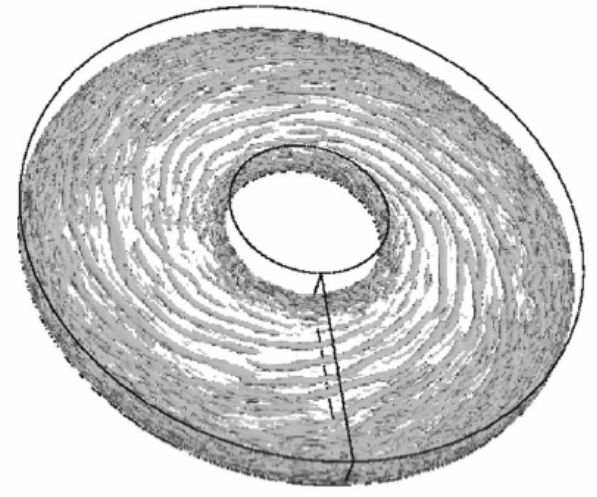

(b)

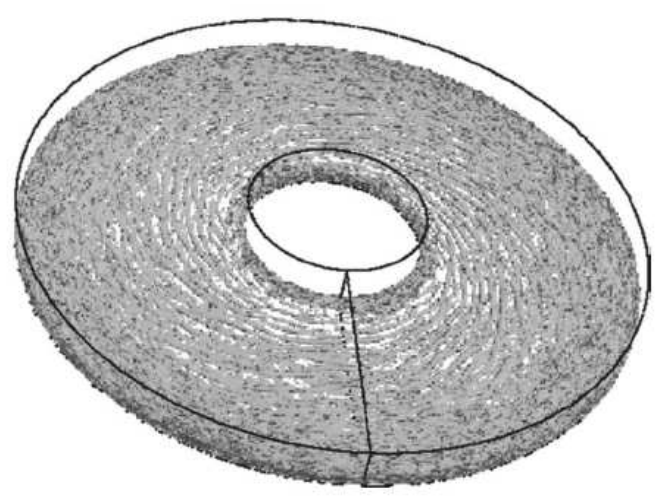

(c)

Figure 10: Launder et al., Annual Rev. Fluid Mech.
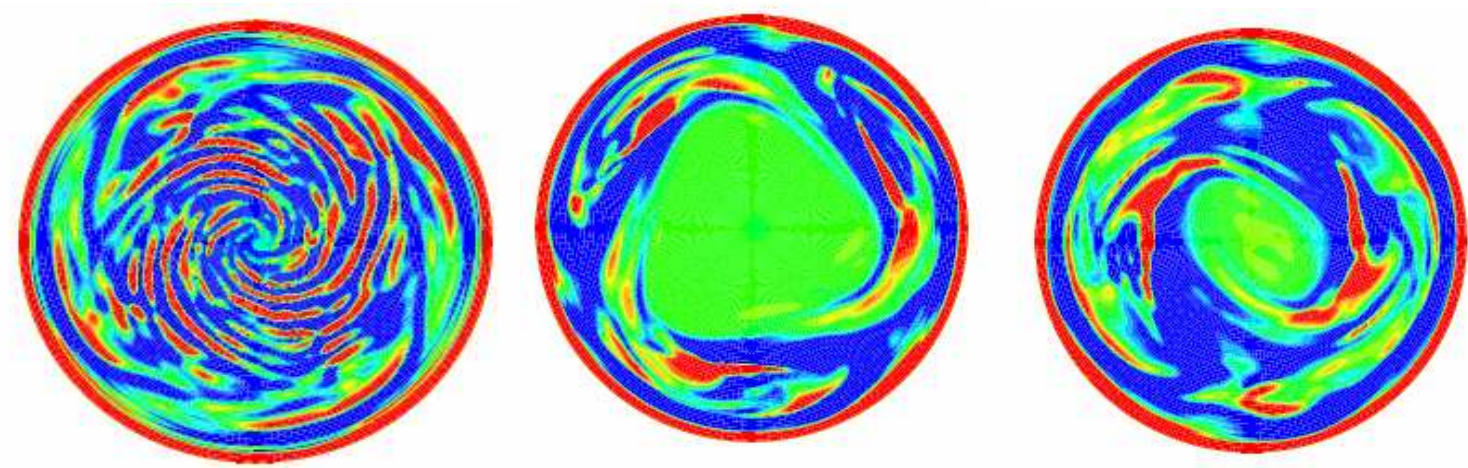

Figure 11: Launder et al., Annual Rev. Fluid Mech. 